\title{
Lattice 2001: Reflections
}

\author{
P. Hasenfratz ${ }^{\mathrm{a}}$

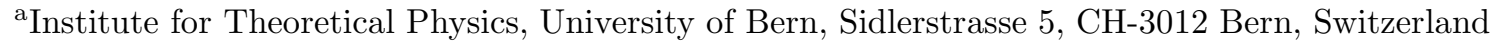

A few subjects which strongly intertwine our field are discussed: $K \rightarrow \pi \pi$ decay, chiral symmetry on the lattice and a few other selected topics. Open questions are touched also on perturbation theory, locality, Gribov copies, $\mathrm{CP}$ symmetry in chiral gauge theories and cut-off effects.

\section{Introduction}

For the final talk of this exciting conference I have chosen a small number of subjects where the progress impressed me, or which dominated the field lately, or simply lie close to my heart.

The first part deals with the heroic work of the CP-PACS and RBC groups on $K \rightarrow \pi \pi$. It is exciting and somewhat frightening to see how brains, software and hardware come together in a highly relevant project which is then pushed to the end (which does not necessarily mean full success).

The second part is on chiral symmetric fermions. This field went through a rapid development during the last few years and attracted many contributions at this conference also.

The third part contains selected topics: topological susceptibility, some remarks on light hadron spectroscopy and a few words on cut-off effects.

In a few cases I will raise questions, discuss issues which keep bugging me. These parts will be separated from the main text. It might be that some of them reflect my ignorance only.

\section{2. $K \rightarrow \pi \pi$ from $K \rightarrow \pi$ and $K \rightarrow 0$ with domain wall fermions}

There are many reasons for discussing this problem here. The $K \rightarrow \pi \pi$ decay contains highly relevant physics, it is a very active field of current research and a central problem of our community since the early years of lattice calculations 1 1]. This complex problem is related to exciting theoretical methods and issues like operator product expansion, (quenched) chiral perturbation theory $((Q) \chi P T)$, composite operator renormalization, chiral symmetry, etc.

Interesting possibilities were discussed recently [2,3] to avoid the Maiani-Testa problem[ 国 due to three external particles in the $K \rightarrow \pi \pi$ amplitude. Preliminary results using three-point functions have been presented in Martinelli's plenary talk [5.66]. Here I will discuss the 'reduction method' used by the CP-PACS [7] and RBC groups [8]. In this case the $K \rightarrow \pi \pi$ matrixelement is related to the $K \rightarrow \pi$ and $K \rightarrow 0$ matrixelements 9]. Without a chiral symmetric regularization the problem is not tractable. Both groups used domain wall fermions 10.

The $K \rightarrow \pi \pi$ decay exhibits two significant phenomena of the standard model: the $\triangle I=1 / 2$ rule and the mixing induced and direct $\mathrm{CP}$ violation. The dynamics of these decays is determined by a non-trivial interplay of strong and electroweak forces characterized by several energy scales of very different magnitude from $m_{t}, m_{W}$ to $m_{d}, m_{u}$. At present there is no way to treat all these scales at the same time on the lattice. To disentangle long and short distance contributions operator product expansion is used.

\subsection{Operator product expansion (OPE)}

Consider the $K^{0} \rightarrow \pi^{+} \pi^{-}$transition in leading order of the weak interactions. The quark level transition on the l.h.s. of Fig. 1 is dressed with QCD interactions (the spectator quark is suppressed). Since $M_{W}$ is large relative to the typical momentum $p$ of this problem and so the $W$ boson propagates over a short distance, the four fermion interaction is almost point like. This makes a sys- 


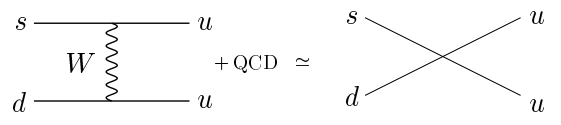

Figure 1. The leading order quark level graph and the corresponding four fermion interaction.

tematic expansion possible for this amplitude:

$$
A=C\left(\mu / M_{W}, \alpha_{s}\right)\langle Q\rangle
$$

with $O\left(p^{2} / M_{W}^{2}\right)$ corrections. QCD effects above the factorization scale $\mu$ are included in the Wilson coefficient $C$, the low energy contributions below $\mu$ are collected into the matrixelement of the local four fermion operators $Q$. This concept of factorization lies at the hart of many QCD applications and I am not aware of reasons to question its applicability.

If $\mu \gg \Lambda_{Q C D}$, we expect that the Wilson coefficient $C\left(\mu / M_{W}, \alpha_{s}\right)$ can be calculated in perturbation theory $(\mathrm{PT})$. The factorization implies that $C$ is independent of the external states and can be calculated using off-shell quarks with high virtuality on both sides of the equation in Fig. 11. The scale $\mu$ has to be chosen judiciously. It should be large enough to justify PT when calculating $C$. On the other hand, if only three light quarks are simulated on the lattice (charm is integrated out), $\mu$ can not be larger than $m_{c} \approx 1.3 \mathrm{GeV}$.

While factorization is a basic assumed feature, the question whether the Wilson coefficients can be calculated perturbatively at a given $\mu / \Lambda_{Q C D}$ is a technical, but important issue.

One can check consistency within PT itself by considering the convergence properties of the expansion, but this is not easy and also not the whole story. With the lattice formulation we have a tool to check the performance of PT by comparing it with the full non-perturbatively calculated result. PT is used in different situations:

i) Physical quantities with a high scale

The number of available well measured short distance physical quantities is very limited. The scale dependence of the running coupling in the Schrödinger functional scheme $\alpha_{S F}(\mu) 11$ is in good agreement with (3-loop) PT up to $\alpha_{S F} \approx 0.3$ in pure Yang-Mills theory 12. Having the $\Lambda$-parameter from this work (in terms of the low energy scale $r_{0} \approx 0.5 \mathrm{fm}[13$ ), PT in Yang-Mills theory is a parameter free expansion. In

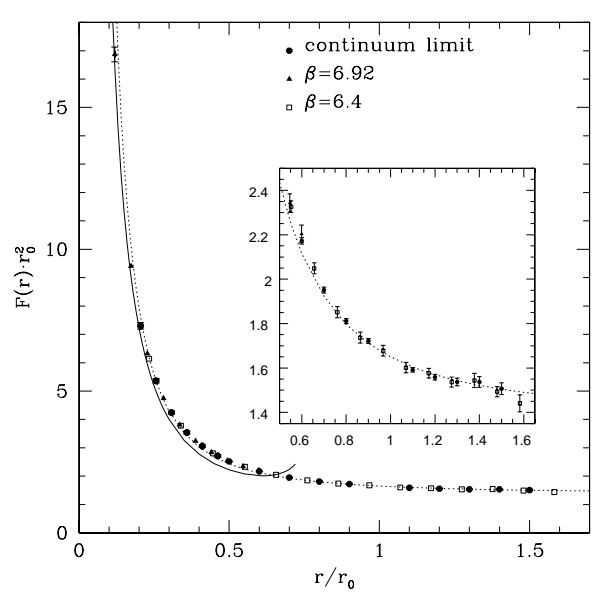

Figure 2. The measured static force compared with PT (solid line) and the string model prediction (dotted line) 14.

a recent work 14 the potential/force was measured at short distances $0.05 \mathrm{fm} \leq r \leq 0.8 \mathrm{fm}$ deep in the continuum limit and the result was compared with PT. The authors pointed out that the perturbative scheme adopted should be chosen judiciously - a point which sheds additional light on the troubled history of PT for the quark potential 15. The scheme defined by the force $\alpha_{q q}(\mu)=r^{2} F(r) / C_{F}, \mu=1 / r$ looks well behaving and describes reasonably the non-perturbative data up to $r \approx 0.15 \mathrm{fm} \approx(1.3 \mathrm{GeV})^{-1}$. Fig. 2 shows another well known feature which is, however, difficult to understand. Including the leading bosonic string model correction in the force $F(r)=$ $\sigma+\pi / 12 r^{2}$ 16, which is expected to work at large distances, describes the data well at quite short distances also. Actually, even at $r \approx 0.15 \mathrm{fm}$ this form is closer to the data than PT. The picture is confusing since, even at the largest distance of this analysis $(1.6 \mathrm{fm})$ the spectrum of the effective QCD string deviates significantly from that of an effective bosonic string 17].

ii) Renormalization constants $Z$

The scale dependent renormalization constants of 


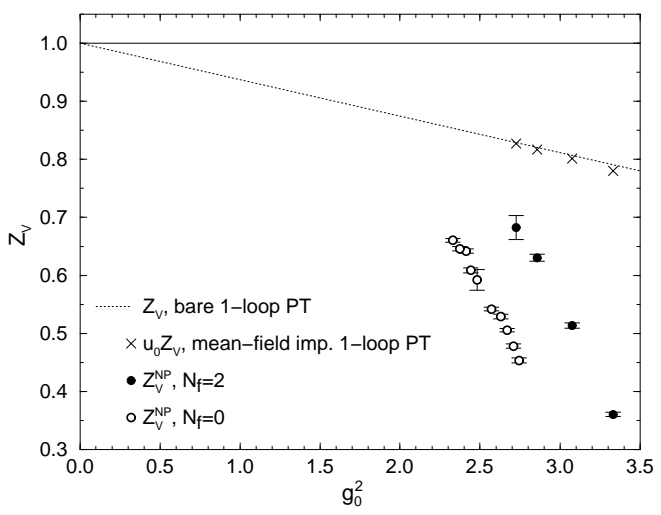

Figure 3. The $Z$-factor of the vector current 22].

composite operators depend on the cut-off and on the renormalization point $\mu$. When combined with renormalization group (RG) this should be a controlled perturbative problem if these scales are large. Nevertheless, as it is well known, PT performs poorly on the renormalization factors. The non-perturbative \{1-loop boosted perturbative\} pseudoscalar renormalization factor $Z_{P}$, for example, at $\mu=2 \mathrm{GeV}$, $1 / a \approx 2 \mathrm{GeV}$ is $0.45(6)\{0.62\}[18,0.39(3)\{0.59\}[19]$ and $0.34\{0.54\} 20$ for Wilson, non-perturbatively improved Wilson and staggered actions, respectively 21. The situation is not always better with the finite renormalization factors, like that of the naive vector current $Z_{V}$. This renormalization factor has no scale $(\mu)$ dependence, it is a function of the lattice coupling $g_{0}$ and goes to 1 in the continuum limit. Fig. 3, taken from 22 1, contains quenched $a \in 0.11-0.20 \mathrm{fm}$ and $N_{f}=2 a \in 0.11-0.23 \mathrm{fm}$ data (RG improved gauge action [24], mean field improved clover quark action) compared with bare and meanfield improved PT.

iii) Cut-off effects, improvement coefficients In order to eliminate the leading cut-off effects (in spectral quantities) the action is extended by a term $C_{S W}\left(g_{0}\right) Q$, where $Q$ is the Sheikholeslami-Wohlert operator 25], while $C_{S W}$ is the Symanzik improvement coefficient 26]. The improvement should work for any external states, also for those with high virtuality, which can be used to calculate the improvement coefficients $C_{S W}\left(g_{0}\right)$. (Notice the analogies with OPE.) For cut-off values of present day simulations $(1 / a \leq 3 \mathrm{GeV})$ PT does not reproduce the non-

\footnotetext{
${ }^{1} \mathrm{I}$ am indebted to Tomoteru Yoshie for a correspondence concerning the results on $Z_{V}$ in 22] and 23.
}

perturbatively fixed $C_{S W}\left(g_{0}\right)$ 27]. For further examples of problems with PT when calculating operator improvement coefficients see Table I in 28.

The conclusion is that in the very few cases where PT was systematically compared with numerical results on physical quantities a judiciously chosen PT scheme seems to work well down to such low scales as $\mu \approx 1.3 \mathrm{GeV}$. On the other hand, in problems where the cut-off also plays a role PT performs poorly for $1 / a \leq 3 \mathrm{GeV}$. Many believe that this is related to some special feature of lattice $\mathrm{PT}$, like the existence of tadpole graphs. I do not think that this is the full explanation. First, a lot of effort was invested to overcome this problem of lattice PT 29. Second, more importantly, some of the quantities in question, do not look like perturbative at all. The $a$ dependence of the coupling $g_{0}$ of the plaquette action (lattice $\beta$ function) [30, the $g_{0}$ dependence of $C_{S W}$ [27], or $Z_{V}$ in Fig. 3, for example, simply do not invite to use PT at all.

The graph in Fig. 1 actually generates two fourfermion operators with different color structure

$$
\begin{aligned}
& Q_{1}=\left(\bar{s}_{a} u_{b}\right)_{L}\left(\bar{u}_{b} d_{a}\right)_{L}, \\
& Q_{2}=\left(\bar{s}_{a} u_{a}\right)_{L}\left(\bar{u}_{b} d_{b}\right)_{L},
\end{aligned}
$$

where $a, b$ are color indices and the short-hand notation $\left(\bar{s}_{a} u_{b}\right)_{R / L}=\bar{s}_{a} \gamma_{\mu}\left(1 \pm \gamma_{5}\right) u_{b}$ is used here and below. The sum of these operators (weighted with the Wilson coefficients which include $V_{u s} V_{u d}^{*}$ from the CKM matrix) taken between the incoming kaon and the outgoing pions in isospin $I=0$, or 2 states

$$
\begin{aligned}
& K^{0} \rightarrow(\pi \pi)_{I}=A_{I} \exp \left(i \delta_{I}\right)= \\
& \frac{G_{F}}{\sqrt{2}} V_{u s} V_{u d}^{*} \sum_{i=1}^{2} z_{i}(\mu)\left\langle\pi \pi_{I}\left|Q_{i}\right| K\right\rangle^{\overline{M S}}(\mu),
\end{aligned}
$$

should explain the large ratio

$$
\frac{1}{\omega}=\frac{\operatorname{Re}\left(A_{0}\right)}{\operatorname{Re}\left(A_{2}\right)} \approx 22 .
$$

i.e. the $\Delta=1 / 2$ rule. In eq (3) $\delta_{I}$ is the phase shift from the final state $\pi \pi$ interaction, and $z_{i}(\mu) \propto C_{i}(\mu)$ Wilson coefficients (and the additional ones for $i=3, \ldots, 10$ below) are known in the standard model at next-to-leading order 31. 
On the other hand, the weak interaction part of the 'tree graph' in Fig. 11 does not know about the third generation and is so CP conserving. In order to see $\mathrm{CP}$ violation we have to go beyond this leading approximation and include the QCD penguin and electroweak diagrams of Fig. 6 .
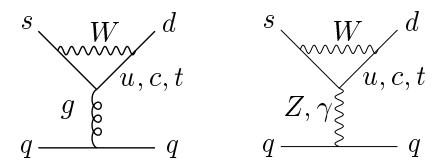

Figure 4. The QCD and electroweak penguins.

The resulting effective hamiltonian is a sum over 10 operators 31. The $\Delta=1 / 2$ problem is expected to be dominated by the $Q_{1}$ and $Q_{2}$ contributions since the functions $z_{i}\left(\mu=m_{c}\right)$ are small for $i=3, \ldots, 10$ [31]. On the other hand, phenomenological considerations suggest [1] that the imaginary part of the amplitude

$$
\begin{aligned}
& \operatorname{Im} A_{I}= \\
& -\operatorname{Im}\left(V_{t s} V_{t d}^{*}\right) \frac{G_{F}}{\sqrt{(2)}} \sum_{i=3}^{10} y_{i}(\mu)\left\langle Q_{i}\right\rangle_{I}^{\overline{M S}}(\mu),
\end{aligned}
$$

where $\left\langle Q_{i}\right\rangle_{I} \exp \left(i \delta_{I}\right)=\left\langle\pi \pi_{I}\left|Q_{i}\right| K\right\rangle$, is dominated by $\left\langle Q_{6}\right\rangle_{I=0}$ and $\left\langle Q_{8}\right\rangle_{I=2}$. In this approximation the direct $\mathrm{CP}$ violation is given by

$$
\begin{aligned}
& \frac{\epsilon^{\prime}}{\epsilon} \approx \frac{\omega G_{F}}{2|\epsilon| \operatorname{Re} A_{0}} \operatorname{Im}\left(V_{t s} V_{t d}^{*}\right) \times \\
& \left(y_{6}(\mu)\left\langle Q_{6}\right\rangle_{I=0}^{\overline{M S}}(\mu)-\frac{1}{\omega} y_{8}(\mu)\left\langle Q_{8}\right\rangle_{I=2}^{\overline{M S}}(\mu)\right)
\end{aligned}
$$

where

$$
\begin{aligned}
& Q_{6}=(\bar{s} d)_{L}\left((\bar{u} u)_{R}+(\bar{d} d)_{R}+(\bar{s} s)_{R}\right), \\
& Q_{8}=\frac{1}{2}(\bar{s} d)_{L}\left((2 \bar{u} u)_{R}-(\bar{d} d)_{R}-(\bar{s} s)_{R}\right) .
\end{aligned}
$$

It is reassuring that this qualitative expectation is supported by the numerical data [7, ह]. Although the contribution from $Q_{8}$ is suppressed by the electroweak coupling constant, this is largely compensated by the prefactor $1 / \omega$ leading to a numerically unpleasant cancellation between the two terms in eq. (6).

\subsection{Determination of the bare $\left\langle\pi \pi_{I}\left|Q_{i}\right| K^{0}\right\rangle$ matrixelements}

The CP-PACS and RBC collaborations followed the 'reduction method', where tree level $\chi P T$ is used to relate the $K^{0} \rightarrow \pi \pi$ matrixelements to those of $K^{+} \rightarrow \pi^{+}$and $K \rightarrow$ vacuum [9] which are then calculated on the lattice.

Although the reduction step simplifies the problem significantly, it introduces an unpleasant mixing with a lower dimensional two-quark operator. The $\left(8_{L}, 1_{R}\right)$ operators, like $Q_{6}$ in eq. (7), mix with the two-quark operator ' $\bar{s} d$ ' since the singlet right handed part communicates with the vacuum 9. This $\left(8_{L}, 1_{R}\right)$ operator should be CPS $(\mathrm{CP}+d \leftrightarrow s)$ invariant (as all the $Q_{i}$ operators are) leading to the form for $m_{s} \neq m_{d}$

$$
\begin{aligned}
& Q_{s u b}=\left(m_{s}+m_{d}\right) \bar{s} d-\left(m_{s}-m_{d}\right) \bar{s} \gamma_{5} d \\
& =\partial_{\mu}\left(\frac{m_{s}+m_{d}}{m_{s}-m_{d}} \bar{s} \gamma_{\mu} d-\frac{m_{s}-m_{d}}{m_{s}+m_{d}} \bar{s} \gamma_{\mu} \gamma_{5} d\right) .
\end{aligned}
$$

In eq. (8) the equation of motion was used to write $Q_{s u b}$ as a total divergence for $m_{s} \neq m_{d}$. In the physical $K^{0} \rightarrow \pi \pi$ matrixelement this total divergence does not contribute since the weak operator carries zero momentum. On the other hand, for the $K^{+} \rightarrow \pi^{+}$reduced matrixelement $Q_{\text {sub }}$ gives a (quadratically divergent) contribution: if $m_{s}=m_{d}\left(m_{\pi}=m_{K}\right)$ is used in the simulation (CP-PACS, [7] ) then $Q_{\text {sub }}$ is not a total divergence; if $m_{s} \neq m_{d}$ (RBC, [8]) then the operator carries momentum and the total divergence contributes. This quadratic divergence should be subtracted from the $\triangle I=1 / 2$ operator matrixelements with a coefficient determined by the corresponding $K \rightarrow 0$ matrixelement. The reduction equations have the form $\left(f_{\pi} \approx 93 \mathrm{MeV}\right)$

$$
\begin{aligned}
& \left\langle\pi^{+} \pi^{-}\left|Q_{i}^{\triangle I=1 / 2}\right| K^{0}\right\rangle= \\
& i \frac{m_{K}^{2}-m_{\pi}^{2}}{\sqrt{2} f_{\pi} M^{2}}\left\langle\pi^{+}\left|Q_{i}^{\triangle I=1 / 2}-\alpha_{i} Q_{s u b}\right| K^{+}\right\rangle,
\end{aligned}
$$

for $i=1, \ldots, 6,9,10$ and $\alpha_{i}$ is determined by

$$
\left\langle 0\left|Q_{i}^{\triangle I=1 / 2}-\alpha_{i} Q_{s u b}\right| K^{+}\right\rangle=0 .
$$

The $\triangle I=3 / 2$ operators have no subtractions and for $i=1, \ldots, 6,9,10$ the relations are like in 
eq. (9) with $\alpha_{i}=0$. For $i=7,8$ we have

$$
\left\langle\pi^{+} \pi^{-}\left|Q_{i}^{I}\right| K^{0}\right\rangle=-\frac{1}{\sqrt{2} f_{\pi}}\left\langle\pi^{+}\left|Q_{i}^{I}\right| K^{+}\right\rangle .
$$

In the equations above $m_{K}$ and $m_{\pi}$ are the physical masses, while in the $K \rightarrow \pi$ matrixelement $m_{K}=m_{\pi}=M$. Remarks:

- The reduction is on the tree level of $(\mathrm{Q}) \chi \mathrm{PT}$ only. The $O\left(p^{4}\right)$ low energy constants needed for $K \rightarrow \pi \pi$ can not be found from the $K \rightarrow \pi$, $K \rightarrow 0$ matrixelements. One can use the $O\left(p^{4}\right)$ (Q) $\chi \mathrm{PT}$ results for $K \rightarrow \pi$ [32] to make a more reliable fit for the tree level constants, but even in this case $K \rightarrow \pi \pi$ will be obtained on the tree level only. The 1-loop chiral corrections are estimated to be significant $\left(m_{K} \approx 500 \mathrm{MeV}\right.$ !) [32,33]. - The reduction step relies heavily on chiral symmetry. The mixing problem can not be treated in the $\triangle I=1 / 2$ sector with Wilson fermions.

- Both groups demonstrated that a controlled signal can be obtained after the power divergent subtraction.

- As eq. (9) shows the $K^{+} \rightarrow \pi^{+}$matrixelements should vanish in the chiral limit $M \rightarrow 0$ for $i \neq 7,8$. CP-PACS finds that this is the case within the errors, RBC observes small non-zero intercepts which might be related to the residual chiral symmetry breaking of the domain wall fermions. As eq. (9) shows, the slope is all what one needs.

\subsection{Renormalization}

CP-PACS used renormalization factors calculated in 1-loop PT 34]. RBC applied the Roma-Southampton non-perturbative method with gauge fixing 35 .

The uncontrolled effect of Gribov copies in the gauge fixing is a frequently discussed issue in this nonperturbative renormalization method. Undoubtedly, it would be very difficult to perform an unbiased averaging over the Gribov copies 36. On the other hand, is such averaging really needed if we study a short distance $(\propto 1 / \mu)$ problem? Is it consistent to worry about Gribov copies and, at the same time accepting the validity of $\mathrm{PT}$ at the scale $\mu$ (say, in $\overline{M S}$ ), which knows nothing about such non-perturbative features?

\subsection{Physical results}

The parameters of the simulations of CP-PACS and $\mathrm{RBC}$ are quite similar. On the real part of $A_{I}$ and so on the $\triangle I=1 / 2$ rule, $\mathrm{RBC}$ obtained results close to those in experiments, while the results on $\operatorname{Re} A_{0}$ and $\omega^{-1}$ from CP-PACS are a factor of $\sim 2$ smaller.

Both groups obtained a small negative $O\left(10^{-4}\right)$ number for $\epsilon^{\prime} / \epsilon$ as opposed to the experimental average of $(17.2 \pm 1.8) 10^{-4}[37]$.

Given the large theoretical and numerical complexity of the problem I find these works very impressive. There are several possible sources of systematical error, like quenching, large corrections to tree level reduction in $\chi \mathrm{PT}$ and (continuum) PT at scales $\sim 1.3 \mathrm{GeV}$. In $[38$ the authors argue that a new low energy constant (a pure quenching artifact) enters the reduction relations which might have an influence on the results above.

\section{Chiral symmetric lattice fermions}

We had four plenary talks at this conference which were directly on this subject[39], or were closely related [40]. I would like to discuss here a few points only 41 .

The Ginsparg-Wilson (GW) relation 42] was suggested as the 'mildest way' to break chiral symmetry on the lattice in 1982 immediately after the no-go theorem of Nielsen and Ninomiya 433. In its simplest form the GW relation reads

$$
\gamma_{5} D+D \gamma_{5}=D \gamma_{5} D
$$

or, equivalently (if $D$ has no zero modes)

$$
\gamma_{5} D^{-1}\left(x, x^{\prime}\right)+D^{-1}\left(x, x^{\prime}\right) \gamma_{5}=\gamma_{5} \delta_{x, x^{\prime}} .
$$

Although the propagator $D^{-1}$ does not anticommute with $\gamma_{5}$ the violation is a contact $\sim \delta_{x, x^{\prime}}$ term only. It is expected and is really so that the GW relation implies chiral symmetry on physical predictions 44.

Eq. (11) is a non-linear relation for $D$. It is clear intuitively and has been shown rigorously 45 that the solution can not be ultralocal, it has a tail. On the other hand, a physically interesting solution should be local, which means that this tail of $D$ should decay faster than the 
signal at physical distances in correlation functions

$$
D\left(x, x^{\prime}\right) \sim \exp \left(-M a\left|x-x^{\prime}\right|\right)_{\left|x-x^{\prime}\right| \gg 1},
$$

where $M=O$ (cut-off).

Let me discuss the important issue of locality of the action in a QFT further. Locality is related to universality and universality implies predictive power. If, however, the action has couplings between distant points which compete with the real dynamics of the system, then the predictions will depend on the nonphysical microscopical details. Universality will be lost and such an action is not useful.

For this reason, the checks concerning the locality of the GW type Dirac operators are very important 39,46 49. Dynamical staggered fermions with $N_{f}<4$ present a not fully understood, potentially dangerous situation. It is not obvious that $\left(\operatorname{det} D(U)_{s t}\right)^{1 / 2}$ in the path integral defines a local theory, although there are no arguments excluding this either 50]. Two dimensional models might be the right place for testing.

Since no solution to the GW relation eq. (11) was found in the presence of gauge fields, the idea was soon after its birth abandoned. The first lattice regularization of fermions with chiral symmetry, the domain wall fermions 10 and the related overlap construction 51 followed a different path and seemed to be unrelated to the GW relation.

Following the observation that the fixed-point Dirac operator satisfies the GW relation 52 the interest turned to this general formulation again. The GW relation is a powerful theoretical tool. Its immediate consequence is the index theorem on the lattice [53] and it implies the existence of an exact chiral symmetry transformation 54]. The observation that the overlap Dirac operator satisfies the GW relation connected the domain wall approach to this general formulation 55.

As the previously discussed $K \rightarrow \pi \pi$ studies illustrate, domain wall and, to a lesser extent, overlap fermions are used today in large scale quenched simulations in problems, where chiral symmetry plays an important role 56, 7, 8, 8].

The fixed-point gauge and Dirac actions are defined by classical equations 57]. These equations can be solved numerically in an iterative way which is, however, far too expensive in a stochastic calculation. A parametrization in terms of a finite number of operators is unavoidable. This can be done quite effectively and the Dirac operator obtained this way performs well in different test runs [58,48]. An alternative way to fix the couplings in a parametrized Dirac operator is a systematic expansion of the GW equation itself [59]. For test results on this action and for comparison with other formulations, see 60.

The new chiral symmetric actions allow to investigate the role of topological excitations in the QCD vacuum. It was convincingly demonstrated in several contributions at this conference that an instanton dominated picture of low eigenmodes of the Dirac operator is consistent: the local peaks in these eigenmodes are dominantly chiral 61.60]. Such a study is hardly possible with Wilson fermions [62].

Chiral symmetric Dirac operators have nice theoretical properties including the absence of exceptional configurations and $O(a)$ improvement in spectroscopy. They are, however, expensive to simulate. The first studies on spectroscopy are exploratory [56, 63 65, 58]. From these works it is obvious that light hadron spectroscopy becomes much cleaner: quark mass tuning and long chiral extrapolations are not needed, topology is well defined and operator renormalization is significantly simplified. The first quantitative results confirm that there are no unexpected, hidden problems with this regularization of QCD. Simulations in the Schwinger model [66] strengthen this conclusion.

There are problems, like the fermion condensate, where a chiral symmetric action is a prerequisite for a quantitative study. The quark condensate, or more precisely, the leading low energy constant $\Sigma$ in the chiral Lagrangian, has been calculated by several groups using different actions and methods 67, 64, 48, The results are consistent and scatter around $\left(\Sigma^{\overline{M S}}(2 \mathrm{GeV})\right)^{1 / 3}=270 \mathrm{MeV}$ with a few percent statistical error. Since no continuum extrapolation is done yet, the systematical error is unknown.

As mentioned before, eq. (11) implies exact 
symmetry under the transformation [54]

$$
\begin{aligned}
& \psi \rightarrow \psi+i \epsilon T^{a} \gamma_{5}(1-D) \psi, \\
& \bar{\psi} \rightarrow \bar{\psi}+i \epsilon \bar{\psi} \gamma_{5} T^{a},
\end{aligned}
$$

$a=1, \ldots, N_{f}^{2}-1$, which is a sort of smeared $\gamma_{5}$ transformation. The action is also invariant under a singlet chiral transformation, but the fermion measure is not (notice that the transformation in eq. (14) is gauge field dependent) leading to the correct anomalous Ward identity

$$
\langle\delta \mathcal{O}\rangle_{F}-2 N_{f} \nu(U)\langle\mathcal{O}\rangle_{F}=0,
$$

where $\nu(U)$ is the topological charge, while $\langle.\rangle_{F}$ is the fermionic path integral on a fixed gauge field configuration $U$.

The transformation in eq. (14) is asymmetric on $\psi$ and $\bar{\psi}$, and so are the left/right projectors also 41.

$$
\psi_{L / R}=\hat{P}_{L / R} \psi, \quad \bar{\psi}_{L / R}=\bar{\psi} P_{R / L},
$$

where

$$
\begin{aligned}
& \hat{P}_{R / L}=\frac{1}{2}\left(1 \pm \hat{\gamma}_{5}\right), \quad P_{R / L}=\frac{1}{2}\left(1 \pm \gamma_{5}\right), \\
& \hat{\gamma}_{5}=\gamma_{5}(1-D) .
\end{aligned}
$$

The fermion action falls into $L$ and $R$ parts as in the formal continuum

$$
\bar{\psi} D \psi=\bar{\psi}_{L} D \psi_{L}+\bar{\psi}_{R} D \psi_{R} .
$$

Eqs. (16,17,18) are the first, almost trivial steps in a highly complex construction which led to a breakthrough concerning chiral gauge theories 68. For anomaly free complex representations for $U(1)$, or $S U(2) \times U(1)$ gauge groups the theory is constructed non-perturbatively 69, 70, for a general compact group it is constructed to all orders of PT[71].

At present, chiral gauge theories obtained this way have a certain ambiguity which I would like to discuss briefly. A normalization factor (absolute value and phase) in $Z_{\nu}$, where $Z_{\nu}$ is the partition function of the theory in the topological sector with charge $\nu$, is left undetermined. This is an unsatisfactory situation since, among others, it leaves the magnitude of fermion violating processes undetermined.

In a recent paper 72 H. Suzuki considered Weyl fermions in a real representation of the gauge group. He demonstrated that this Weyl fermion is equivalent to a Mayorana ( $L-R$ symmetric) fermion on the lattice, like in the continuum. Based on this observation Suzuki suggested a 'natural' relative normalization of different topological sectors for Weyl fermions in complex representations.

The normalization of different topological sectors is related to $\mathrm{CP}$ symmetry in chiral gauge theories a problem which keeps bugging me since 1998 . The present formulation is not explicitly CP symmetric. It might turn out that the renormalized final physical predictions, which include the relative normalizations between different topological sectors, respect CP. If this is the case, the existence of an explicit $\mathrm{CP}$ invariant lattice formulation is not excluded and would be interesting to find. If CP symmetry can not be restored ( $\mathrm{CP}$ anomaly), that would be even more interesting.

Under $\mathrm{CP}, \psi \rightarrow \bar{\psi} W, \bar{\psi} \rightarrow-W^{-1} \psi, U \rightarrow$ $U^{\mathrm{CP}}$, the vector gauge theory is invariant if $W D\left(U^{\mathrm{CP}}\right)^{T} W^{-1}=D(U)$, where $U^{\mathrm{CP}}$ is the CPtransformed gauge configuration and in my convention $W=\gamma_{2}$. $\mathrm{I}$ assume that $D$ satisfies this condition. However, unlike in the continuum, the left handed part of the fermion action is not invariant:

$$
\bar{\psi}_{L} D \psi_{L} \stackrel{\mathrm{CP}}{\longrightarrow} \bar{\psi}_{L} D \psi_{L}-\frac{1}{2} \bar{\psi} D \gamma_{5} D \psi .
$$

One might try to solve this problem by observing that the choice for the projection operators in eq. (17) is not unique. One can find a continuous set of projectors constructed in terms of $\Gamma_{5}^{(s)}=\gamma_{5}(1-s D) / \mathcal{N}^{(s)}$ and $\overline{\Gamma_{5}^{(s)}}=(1-(1-s) D) \gamma_{5} / \mathcal{N}^{(s)}$ for the fermions and antifermions, respectively, where $s$ is an arbitrary real parameter and $\mathcal{N}^{(s)}$ is for the correct normalization. These projectors assure the $L+R$ decomposition of eq. (18) and give $\nu$ for the difference between the dimension of the left handed fermion and antifermion

\footnotetext{
${ }^{2}$ Martin Lüscher raised my attention to the fact that the projection operators $\hat{P}_{R / L}$ in eq. 117) do not follow the continuum transformation laws under $\mathrm{CP}$.

${ }^{3}$ Under CP the spatial coordinates are reflected which we keep implicit in the following equations.
} 
spaces $4^{1}$ for any value of $s$. On the other hand, the CP violating term in eq. (19) will be proportional to $1-2 s$ and so for $s=1 / 2$ the left handed action becomes CP invariant. Unfortunately, just at this value of $s$ the projectors become singular (non-local). It seems to be difficult to define a CP invariant left handed action.

Return back to the original definition in eq. (17) and consider the fermionic expectation value on the gauge field configuration $U$

$\langle\mathcal{O}\rangle_{F}=K_{\nu} \int D \psi_{L} D \bar{\psi}_{L} \exp \left(-\bar{\psi}_{L} D(U) \psi_{L}\right) \mathcal{O}$,

where $\mathcal{O}$ is some expression of the left handed fields and $K_{\nu}$ is the unknown normalization factor of the topological sector $\nu$. Introducing a basis for the fermions and antifermions

$$
\begin{array}{ll}
\hat{P}_{L} v_{j}=v_{j}, & \psi_{L}(x)=\sum_{j} c_{j} v_{j}(x), \\
P_{R} \omega_{k}=\omega_{k}, & \bar{\psi}_{L}(x)=\sum_{k} \bar{c}_{k} \omega_{k}^{\dagger},
\end{array}
$$

eq. (20) can be written as

$$
\begin{aligned}
\langle\mathcal{O}\rangle_{F}= & K_{\nu} \prod_{j, k} \int d c_{j} \int d \bar{c}_{k} \\
& \exp \left(-\sum_{k, j} \bar{c}_{k} M_{k, j} c_{j}\right) \mathcal{O},
\end{aligned}
$$

where $M_{k, j}=\left(\omega_{k}, D v_{j}\right)$. Going over to a different basis, the measure changes by a gauge field dependent phase. The difficult part of defining a chiral gauge theory is to fix this phase (or the basis) so that the final theory is local and gauge invariant 6871 .

The absolute value $\left|\langle\mathcal{O}\rangle_{F}\right|$ is, however, independent of the basis chosen. One might construct the $\left\{v_{j}\right\}$ and $\left\{\omega_{k}\right\}$ basis vectors in eq. (21) in terms of the eigenvectors of $D$, for example.

Consider a gauge field configuration $U\left(U^{\mathrm{CP}}\right)$ with topological charge $\nu=1(\nu=-1) . D(U)$ has two left handed fermions with $\lambda=0$ and 2, further a $\lambda=2$ left handed antifermion. $D\left(U^{\mathrm{CP}}\right)$ has a left handed antifermion with $\lambda=0$ and no left handed modes with $\lambda=2$. The complex eigenvalues on $U$ and $U^{\mathrm{CP}}$ are the same.

Let us chose a simple operator $\mathcal{O}=c_{0}=\left(u_{0}, \psi\right)$, where $u_{0}$ is the zero mode $D(U) u_{0}=0$. Eq. (22) gives on the gauge field $U$

$$
\left|\langle\mathcal{O}\rangle_{F}\right|=\left|\left\langle c_{0}\right\rangle_{F}\right|=\left|K_{+1}\right| 2 \prod|\lambda|,
$$

\footnotetext{
${ }^{4}$ This is the source of fermion number violation.
}

where every complex pair of eigenvalues $\lambda, \lambda^{*}$ enters the product once. The factor 2 comes from the $\lambda=2$ left handed mode.

Compare eq. (23) with $\left|\langle\overline{\mathcal{O}}\rangle_{F}\right|$ on the configuration $U^{\mathrm{CP}}$, where $\overline{\mathcal{O}}=\mathcal{O}^{\mathrm{CP}}=\left(\bar{\psi}, s_{0}\right)$ and $s_{0}$ is the right handed zero mode on $U^{\mathrm{CP}}$. One obtains

$$
\left|\left\langle\mathcal{O}^{\mathrm{CP}}\right\rangle_{F}\right|=\left|K_{-1}\right| \prod|\lambda|
$$

In eq. (24) the factor of 2 is missing: on $U^{\mathrm{CP}}$ with $\nu=-1$ there is no left handed mode with $\lambda=2$.

Suzuki, using a consideration unrelated to CP symmetry, suggested a 'natural' relative normalization $K_{\nu}=2^{-\nu / 2}$ [72. This factor would restore CP symmetry in the absolute value of the expectation values above. The question remains, however, whether this choice is consistent with cluster decomposition in the case where an instanton and anti-instanton are separated in a $\nu=0$ configuration.

\section{Selected topics}

\subsection{The topological susceptibility}

The topological susceptibility

$$
\chi=\left\langle\int d^{4} x q(x) q(0)\right\rangle=\frac{\left\langle\nu^{2}\right\rangle}{V},
$$

where $q(x)$ is the topological charge density and $\nu=\int d^{4} x q(x)$. This quantity is related to one of the fanciest features of QCD, like the $U(1)$ problem, the axial anomaly and their interplay with topology.

The topological susceptibility $\chi^{q}$ calculated in quenched QCD (i.e. over configurations generated with the Yang-Mills action) enters the Witten-Veneziano relation 73

$$
\chi^{q}=\frac{f_{\pi}^{2} m_{\eta^{\prime}}^{2}}{2 N_{f}}, \quad N_{c} \rightarrow \infty
$$

in the chiral limit. In full QCD, configurations with non-zero topological charge $\nu$ are suppressed in the chiral limit by the fermion determinant. The leading correction to the chiral limit reads 74,75

$$
\chi=\frac{m_{q} \Sigma}{N_{f}}=\frac{f_{\pi}^{2} m_{\pi}^{2}}{2 N_{f}},
$$


where $-\Sigma=\langle\bar{u} u\rangle=\langle\bar{d} d\rangle \ldots$ is the quark condensate (in our normalization $f_{\pi} \approx 93 \mathrm{MeV}$ in $\mathrm{Na}$ ture). For a recent brief review on the history around these relations, see [77], while for a recent summary on numerical simulations of $\chi^{q}$ I refer to [76]. Here I would like to mention some recent developments only.

On the theoretical side the $\mathrm{GW}$ formulation (which has exact $S U\left(N_{f}\right) \times S U\left(N_{f}\right)$ symmetry with the proper $U(1)$ anomaly and the index theorem) allows a clean derivation of eqs. (26,27) on the lattice. Under the standard dynamical assumptions (chiral symmetry is spontaneously broken and the $\eta^{\prime}$ remains heavy) eq. (27) was derived in 75. In a recent paper the WittenVeneziano formula was also reproduced on the lattice [77. It is interesting to notice the analogies between the form of eqs. (26.27) and also in the steps of derivation. Although the $\eta^{\prime}$ mass is due to a hard symmetry breaking (anomaly), it is switched off by the $N_{c} \rightarrow \infty$ limit similarly as the soft breaking $m_{q}$ is switched off in the chiral limit.

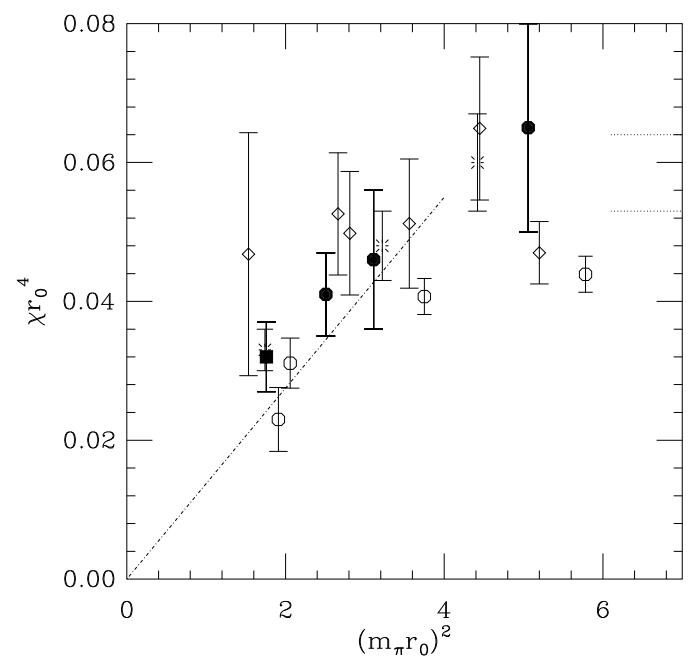

Figure 5. $N_{f}=2$ data: Wilson fermions 82 (open diamond), clover fermions 81. (open circle), staggered fermions with smeared link operator 85.(filled symbols), clover fermions with RG improved action [88](burst).
On the numerical side, the quenched topological susceptibility seems to settle down at a value $\chi^{q}=(0.205 \mathrm{MeV})^{4}$ with an estimated $10 \%$ error on $\chi^{q}$ [78.76]. A specialty of the topological susceptibility is that its cut-off effects depend not only on the action but also on the details of the calculation (type and number of cooling steps) if non-chiral symmetric actions with a 'field theoretic' definition of $\nu$ are used. The value above compares reasonably with eq. (26) if $N_{c}=3$ numbers are inserted on the r.h.s.. $N_{c}=3$ seems to be close to the $N_{c} \rightarrow \infty$ limit which is also supported by a recent numerical study of the string tension, glue ball masses and $\chi^{q}$ in $S U(N), 2 \leq N \leq 5[79]$. The numerical analysis becomes cleaner if chiral symmetric actions are simulated. Only preliminary results are available 80.48 .

The topological susceptibility of QCD is, presumably the best place to see, how sea quarks turn Yang-Mills gauge theory in full QCD. As eq. (27) shows, the effect should be striking: the susceptibility $\chi$ goes to zero in the chiral limit. The early results 8183 appear controversial. The authors in ref. 85,86 argue that the cutoff effects are responsible for the confusing results, in particular, chiral symmetry violation for Wilson type fermions and flavor symmetry violation for staggered fermions 85. at large lattice spacings. The situation is expected to become better at smaller $a$, with improved actions, or improved topological charge operators. This expectation is confirmed by the $N_{f}=2$ CP-PACS 88 and by the results in Fig. 国.

Fig. is a compilation 85 of staggered, Wilson and clover data with $N_{f}=2$ at $a \approx 0.1 \mathrm{fm}$, where, with the exception of the SESAM data [82], either the action, or the operator is improved. The effect of dynamical fermions is seen clearly and the data are consistent with $\chi \rightarrow 0$ as $m_{\pi} \rightarrow 0$. On the other hand, remaining discretization errors might be significant and the behavior of $\chi$ with respect to $\left(m_{\pi} r_{0}\right)^{2}$ might look differently in the continuum limit 87.

\subsection{On quenched spectroscopy}

Quenched QCD is not a healthy QFT, but believed to be universal in the continuum limit. The data are not very convincing, but at least do not 
contradict this expectation.

In my talk I referred to Sinya Aoki's contribution in Bangalore 89, where the strong disagreement between the staggered and Wilson Edinburgh plots was discussed. The situation concerning the hyperfine splittings confused me also. By now I understand these points better.

'The staggered and Wilson fermion Edinburgh plots strongly disagree'. I believe, the reason of the disagreement in Fig.4 in 89 are the very large cut-off effects in the unimproved staggered data which made a continuum extrapolation unreliable. This is nicely demonstrated in Fig.19 of ref. 90 which shows that by reducing the cut-off effects in the staggered simulations (by simulating at small $a$, like in 91, or by using improved fermions) the staggered Edinburgh plot is pushed towards to that obtained with Wilson type of fermions.

'Are the quenched hyperfine splittings too small, or too large?' They were found too small by many groups and most convincingly by the CP-PACS Collaboration 92. On the other hand, UKQCD finds the hyperfine splittings always far too large 93. I think the reason is that UKQCD fixed the scale from $r_{0} \approx 0.5 \mathrm{fm}$, while the standard choice is fixing $m_{\rho}$. Fixing $m_{\rho}$ gives $r_{0} \approx 0.55 \mathrm{fm}$ (see, for example 22 ) and this $10 \%$ effect explains the paradox with the hyperfine splittings. The disagreement of quenched spectroscopy with Nature can be shifted to different quantities by changing the method of fixing the scale.

\subsection{Strange cut-off effects}

Numerical data in asymptotically free theories are standardly extrapolated to the continuum using Symanzik's results 26] valid in every order of perturbation theory. Accordingly, in a bosonic theory the leading cut-off effect is quadratic $O\left(a^{2}\right)$ modified by logarithms. Results in the $O(3)$ non-linear sigma model show that either nonperturbative effects invalidate this expectation, or the onset of leading behavior happens at very large correlation lengths only 94. In Fig. 6 the data obtained with two different actions on the step scaling function 95] follow a linear $O(a)$ type of behavior - at least up to correlation lengths 350. The cut-off dependence of the zero momentum 4-point function is similar 94,95. One should be open minded.

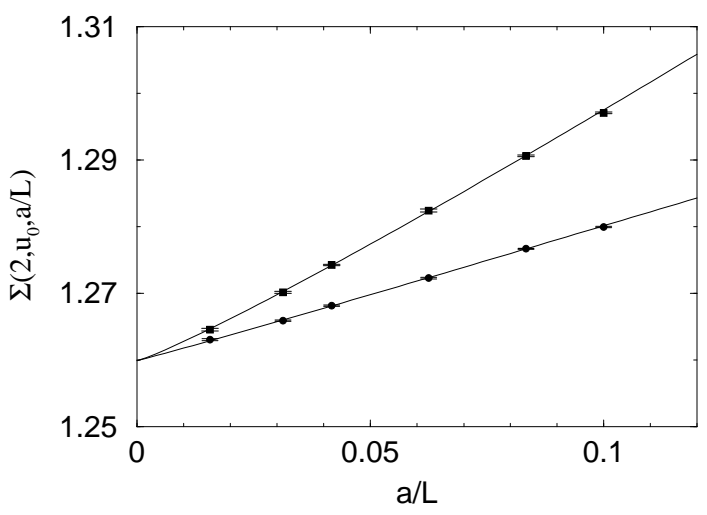

Figure 6 . The step scaling function $\Sigma\left(2, u_{0}, a / L\right)$ at $u_{0}=1.0595$ for two different actions.

Acknowledgements: I am indebted to Claude Bernard, Gilberto Colangelo, Tom DeGrand, Christof Gattringer, Maarten Golterman, Anna Hasenfratz, Julius Kuti, Christian Lang, Guido Martinelli, Steve Sharpe and my colleagues in Bern for useful discussions. I thank for the kind hospitality during a workshop at the Institute for Nuclear Theory, Univ. Washington, where part of this work was completed.

This work has been supported in part by the Schweizerischer Nationalfonds and the European Community's Human Potential Programme under contract HPRN-CT-2000-00145.

\section{REFERENCES}

1. G. Buchalla, hep-ph/0103166; C. Bernard and A. Soni, Nucl. Phys. B(Proc. Suppl.) 9 (1989) 153; L. Lellouch, Nucl. Phys. B(Proc. Suppl.) 94 (2001) 142.

2. L. Lellouch and M. Lüscher, Commun. Math. Phys. 219 (2001) 31.

3. C.-J. D. Lin et al., hep-lat/0104006.

4. L. Maiani and M. Testa, Phys. Lett. B245 (1990) 585.

5. G. Martinelli, these proceedings. 
6. Ph. Boucoud et al., SPQR, hep-lat/0110169, hep-lat/0110206.

7. J. Noaki et al., CP-PACS, hep-lat/0108013, hep-lat/0110142.

8. T. Blum et al., RBC, hep-lat/0110075, heplat/0110175, hep-lat/0110144.

9. C. Bernard et al., Phys. Rev. D32 (1985) 2343.

10. D. B. Kaplan, Phys. Lett. B288 (1992) 342; Y. Shamir, Nucl. Phys. B406 (1993) 90; V. Furman and Y. Shamir, Nucl. Phys. B439 (1995) 265.

11. M. Lüscher et al., Nucl. Phys. B389 (1993) 247, ibid B413 (1994) 481.

12. S. Capitani et al., ALPHA, Nucl. Phys. B544 (1999) 669.

13. R. Sommer, Nucl. Phys. B411 (1994) 839.

14. S. Necco and R. Sommer, hep-lat/0108008, hep-ph/0109093.

15. A. Billoire, Phys. Lett. B92 (1986) 343; M. Peter, Nucl. Phys. B501 (1997) 471;

Y. Schröder, Phys. Lett. B447 (1999) 321;

M. Melles, Phys. Rev D62 (2000) 074019.

16. M. Lüscher, Nucl. Phys. B180 (1981) 317.

17. K. J. Juge, J. Kuti and C. Morningstar, heplat/0103008; J. Kuti, hep-lat/0110157.

18. V. Gimenez et al. Nucl.Phys.B531 (1998) 429.

19. D. Becirevic et al., Phys. Lett. B444 (1998) 401.

20. S. Aoki et al., Nucl. Phys. (Proc. Suppl)73 (1999) 279

21. S. R. Sharpe, hep-lat/9811006.

22. A. A. Khan et al. CP-PACS, hep-lat/0105015.

23. S. Aoki et al, CP-PACS, hep-lat/0110128.

24. Y. Iwasaki, Nucl. Phys. B258 (1985) 141.

25. B. Sheikholeslami, R. Wohlert, Nucl. Phys. B259 (1985) 572.

26. K. Symanzik, Nucl. Phys. B226 (1983) 187, 205.

27. M. Lüscher et al.,ALPHA, Nucl. Phys. B478 (1996) 365, ibid B491 (1997) 323.

28. T. Bhattacharya et al., hep-lat/0111001.

29. G. P. Lepage and P. Mackenzie, Phys. Rev. D48 (1993) 2250.

30. K. C. Bowler et al., Nucl. Phys. B257(FS14) (1985) 155; K. C. Bowler et al., Phys. Lett. B163 (1985) 367.

31. for a review, see G. Buchalla, A. J. Buras and M. E. Lautenbacher, Rev. Mod. Phys. 68 (1996) 1125; A. J. Buras, hep-ph/0101336.

32. M. Golterman and E. Pallante, JHEP 0008 (2000) 023, Nucl. Phys. B(Proc. Suppl.)83-84 (2000) 250, M. Golterman, hep-ph/0011084.

33. G. Colangelo, hep-lat/0111003; G. Colangelo, J. Gasser and H. Leutwyler, Nucl. Phys. B603 (2001) 125.

34. S. Aoki et al., Phys. Rev. D59 (1999) 094505, ibid D60 (1999) 114504, S. Aoki and Y. Kuramashi, Phys. Rev. D63 (2001) 054504.

35. G. Martinelli et al., Nucl. Phys. B445 (1995) 81.

36. for a review, see L. Giusti et al., Int. J. Mod. Phys. A16 (2001) 3487; O. Oliveira and P. J. Silva, hep-lat/0110035.

37. A. Alavi-Harati et al., KTEV, Phys. Rev. Lett.83 (1999) 22; V. Fanti et al., NA48, Phys. Lett. B465, (1999) 335.

38. M. Golterman and E. Pallante, heplat/0108010.

39. P. Hernandez, hep-lat/0110218; Y. Kikukawa, these proceedings.

40. P. M. Damgaard, hep-lat/0110192; R. G. Edwards, these proceedings.

41. for reviews, see F. Niedermayer, Nucl. Phys (Proc. Suppl.)73 (1999) 105; H. Neuberger, Nucl. Phys. (Proc. Suppl.)83-84 (2000) 67; for a pedagogical overview, see M. Lüscher, hep$\mathrm{ph} / 0102028$.

42. P. H. Ginsparg and K. G. Wilson, Phys. Rev. D25 (1982) 2649.

43. N. B. Nielsen and M. Ninomiya, Phys. Lett. B105 (1981) 219, Nucl. Phys. B185 (1981) 20.

44. P. Hasenfratz, Nucl. Phys. B525 (1998) 401.

45. I. Horvath, Phys. Rev. Lett.81 (1998) 4063, Phys. Rev. D60 (1999)03451; W. Bietenholz, hep-lat/9901005.

46. P. Hernandez, K. Jansen and M. Lüscher, Nucl. Phys. B552 (1999) 363.

47. K. Fujikawa and M. Ishibashi, Nucl. Phys. B605 (2001) 365.

48. P. Hasenfratz et al., hep-lat/0109007.

49. A. Borici, hep-lat/9810064.

50. The author is indebted to Claude Bernard, Maarten Golterman and Steve Sharpe for related discussions.

51. R. Narayanan and H. Neuberger, Phys. Rev. 
Lett.71 (1993) 3251, Nucl. Phys. B412 (1994) 574, ibid B443 (1995) 395; S. Randjbar-Daemi and J. Strathdee, Phys. Lett. B348 (1995) 543, Nucl. Phys. B443 (1995) 386, ibid B466 (1996) 335.

52. P. Hasenfratz, Nucl. Phys. B(Proc. Suppl.)63(A-C) 1998) 53.

53. P. Hasenfratz, V. Laliena and F. Niedermayer, Phys.LettB427 (1998) 125.

54. M. Lüscher, Phys.LettB428 (1998) 342.

55. H. Neuberger, Phys. Lett. B427 (1998) 353; Y. Kikukawa and T. Noguchi, heplat/9902022.

56. T. Blum et al., hep-lat/0007038.

57. P. Hasenfratz and F. Niedermayer, Nucl. Phys. B414 (1994) 785; T. DeGrand et al., Nucl. Phys. B454 (1995) 587; W. Bietenholz et al., Nucl.Phys.(Proc. Suppl.) 53 (1997) 921.

58. P. Hasenfratz et al., hep-lat/0109004.

59. C. Gattringer, Phys. Rev. D63 (2001) 114501; C. Gattringer and I. Hipp, Phys.Lett. B480 (2000) 112; C. Gattringer, I. Hipp and C. B. Lang, Nucl. Phys. B597 (2001) 451.

60. C. Gattringer et al., hep-lat/0110015.

61. T. DeGrand and A. Hasenfratz, heplat/0103002; T. DeGrand, hep-lat/0110005; R. G. Edwards and U. M. Heller, heplat/0105006; C. Gattringer et al., heplat/0105023, hep-lat/0107016; T. Blum et al., hep-lat/0105006.

62. I. Horvath et al., hep-lat/0102003.

63. Y. Aoki, RBC, hep-lat/0110143.

64. L. Giusti, C. Hoelbling and C. Rebbi, heplat/0108007, hep-lat/0110184.

65. S. J. Dong et al., hep-lat/0108020; S. J. Dong et al, hep-lat/0110044.

66. C. B. Lang and T. K. Pani, Nucl. Phys. B513 (1998) 645; L. Giusti, C. Hoelbling and C. Rebbi, Nucl. Phys. (Proc. Suppl.)94 (2001) 741, Phys. Rev. D64 (2001) 054501.

67. P. Hernandez, K. Jansen and L. Lellouch, Phys. Lett. B469 (1999) 198; P. Hernandez et al., JHEP 0107 (2001) 018; T. DeGrand, Phys. Rev. D63 (2001) 034503.

68. for a review,see M. Lüscher, Nucl. Phys. (Proc.Suppl)83 (2000) 34.

69. M. Lüscher, Nucl. Phys. B549 (1999) 295.

70. Y. Kikukawa and Y. Nakayama, Nucl. Phys.
B597 (2001) 519.

71. M. Lüscher, JHEP 06 (2000) 028; H. Suzuki, Nucl. Phys. B585 (2000) 471.

72. H. Suzuki, JHEP 0010 (2000) 039.

73. E. Witten, Nucl. Phys. B156 (1979) 269;

G. Veneziano, Nucl. Phys. B59 (1979) 213, Phys. Lett. B95 (1980) 90.

74. P. Di Vecchia and G. Veneziano, Nucl. Phys. B171 (1980) 253; H. Leutwyler and A. Smilga, Phys. Rev. D46 (1992) 5607).

75. S. Chandrasekharan, Phys. Rev. D60 (1999) 074503.

76. M. Teper, Nucl. Phys. B(Proc. Suppl.) 83-84 (2000) 146.

77. L. Giusti et al., hep-lat/0108009, heplat/0110036.

78. B. Alles, M. D'Elia and A. D. Giacomo, Nucl. Phys. B494 (1997) 281; Ph. de Forcrand et al., Nucl. Phys. B(Proc. Suppl.) 63 (1998) 549; A. Hasenfratz and C. Nieter, Phys. Lett. B439 (1998) 366.

79. B. Lucini and M. Teper, hep-lat/0103027.

80. R.G.Edwards, U.M.Heller and R.Narayanan, Nucl. Phys. B535 (1998) 403.

81. A. Hart and M. Teper, Nucl. Phys. B(Proc. Suppl.) 83 (2000) 746, hep-lat/0009008.

82. G. S. Bali et al. SESAM, hep-lat/0102002.

83. A. A. Khan et al. CP-PACS, Nucl. Phys. B(Proc. Suppl.) 83 (2000) 162.

84. B. Alles, M. D'Elia and A. D. Giacomo, Phys. Lett. B483 (2000) 139, Nucl. Phys. B(Proc. Suppl.) 83 (2000) 431.

85. A. Hasenfratz, hep-lat/0104015.

86. A. Hart and M. Teper, hep-lat/0108006.

87. S. Dürr, hep-lat/0108015.

88. A. A. Khan et al. CP-PACS, hep-lat/0106010.

89. S. Aoki, Nucl. Phys. B(Proc. Suppl.) 94 (2001) 3 .

90. C. Bernard et al. hep-lat/0104002.

91. S. Kim and S. Ohta, hep-lat/9912001.

92. S. Aoki et al., CP-PACS, Phys. Rev. Lett. 84 (200) 238 ;

93. K. C. Bowler et al. UKQCD, Phys. Rev. D62 (2000) 054506; C. R. Alltan et al. UKQCD, hep-lat/0107021.

94. P. Hasenfratz and F. Niedermayer, Nucl. Phys. B596 (2001) 481.

95. M. Hasenbusch et al., hep-lat/0110202. 